Article

\title{
Topological Symmetry Transition between Toroidal and Klein Bottle Graphenic Systems ${ }^{\dagger}$
}

\author{
Mihai V. Putz ${ }^{1,2, *(D)}$ and Ottorino Ori ${ }^{3, *(D)}$ \\ 1 Laboratory of Structural and Computational Physical-Chemistry for Nanosciences and QSAR, \\ Biology-Chemistry Department, Faculty of Chemistry, Biology, Geography, West University of Timisoara, \\ Str. Pestalozzi No. 16, 300115 Timisoara, Romania \\ 2 Laboratory of Renewable Energies-Photovoltaics, R\&D National Institute for Electrochemistry and \\ Condensed Matter, Dr. A. Paunescu Podeanu Str. No. 144, RO-300569 Timisoara, Romania \\ 3 Actinium Chemical Research Institute, Via Casilina 1626/A, 00133 Rome, Italy \\ * Correspondence: mihai.putz@e-uvt.ro or mv_putz@yahoo.com (M.V.P.); ottorino.ori@gmail.com (O.O.) \\ + Paper dedicated in the honor memory of Prof.dr. Mircea V. DIUDEA, a common true friend and an \\ international scholar in mathematical chemistry contributing the nano-topology architecture in special.
}

Received: 15 May 2020; Accepted: 7 July 2020; Published: 27 July 2020

\begin{abstract}
In the current study, distance-based topological invariants, namely the Wiener number and the topological roundness index, were computed for graphenic tori and Klein bottles (named toroidal and Klein bottle fullerenes or polyhexes in the pre-graphene literature) described as closed graphs with $N$ vertices and $3 N / 2$ edges, with $N$ depending on the variable length of the cylindrical edge $L_{C}$ of these nano-structures, which have a constant length $L_{M}$ of the Möbius zigzag edge. The presented results show that Klein bottle cubic graphs are topologically indistinguishable from toroidal lattices with the same size $\left(N, L_{C}, L_{\mathrm{M}}\right)$ over a certain threshold size $L_{C}$. Both nano-structures share the same values of the topological indices that measure graph compactness and roundness, two key topological properties that largely influence lattice stability. Moreover, this newly conjectured topological similarity between the two kinds of graphs transfers the translation invariance typical of the graphenic tori to the Klein bottle polyhexes with size $L_{C} \geq L_{C}$, making these graphs vertex transitive. This means that a traveler jumping on the nodes of these Klein bottle fullerenes is no longer able to distinguish among them by only measuring the chemical distances. This size-induced symmetry transition for Klein bottle cubic graphs represents a relevant topological effect influencing the electronic properties and the theoretical chemical stability of these two families of graphenic nano-systems. The present finding, nonetheless, provides an original argument, with potential future applications, that physical unification theory is possible, starting surprisingly from the nano-chemical topological graphenic space; thus, speculative hypotheses may be drawn, particularly relating to the computational topological unification (that is, complexification) of the quantum many-worlds picture (according to Everett's theory) with the space-curvature sphericity/roundness of general relativity, as is also currently advocated by Wolfram's language unification of matter-physical phenomenology.
\end{abstract}

Keywords: graphenic nano-systems; polyhexes; tori; Klein bottle; topo-quantum symmetry

\section{Introduction}

In 1882, German mathematician Felix Klein (1849-1925) introduced his peculiar "fläshe" (surface), the Klein bottle (KB), several decades following the discovery of the Möbius strip (M) independently made by August Ferdinand Möbius and Johann Listing. These two non-orientable surfaces, with genus $1(\mathrm{M})$ and $2(\mathrm{~KB})$, are deeply interrelated: the Klein surface is obtainable by closing in a cylindrical way the open edges of a Möbius strip. This mechanism explains why the Klein bottle is often defined as 
a surface built by "sewing two Möbius strips together" [1-3] in which the Möbius-halves have the opposite chirality.

The next logical step, i.e., connecting a Möbius-like structure to the open edges of the Möbius strip, leads to the construction of the projective plane, a surface studied by Klein himself in 1874 . Reference [4] provides illustrations of the various forms of interplay among different surfaces (Figure 1).

(A)

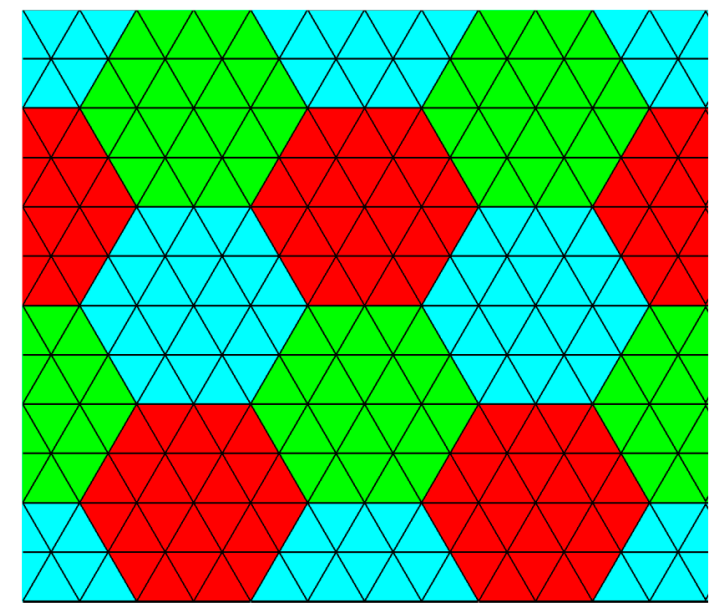

(B)

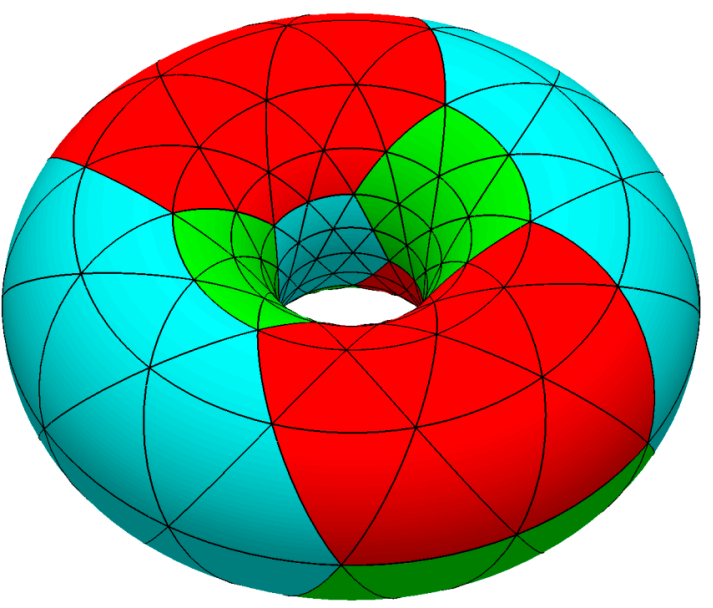

(C)

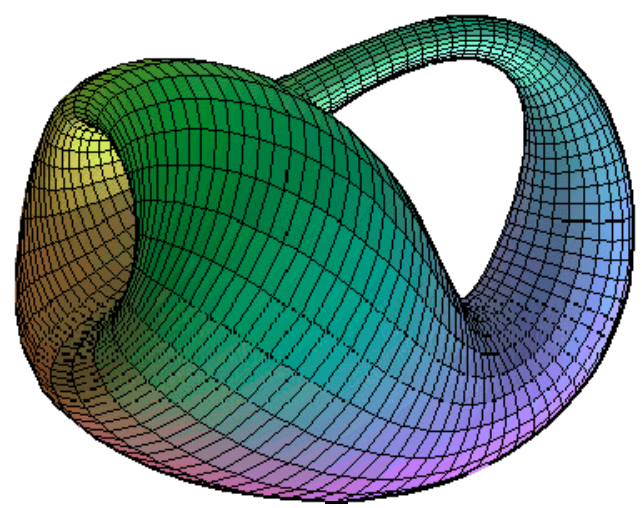

Figure 1. So-called "graphenic nano-structures": (A) the graphene graph as a 2D structure "with boundary"; (B) the torus variety; and (C) the Klein bottle variety, as the 2D manifolds without a boundary; see the text for more details regarding the Möbius strip, see [2]. 
Due to repetition, the word "flasche" (bottle) rapidly came into use, and the surface has since been universally known as the Klein bottle. Indubitably, the KB is a highly attractive topological structure, not only for theoretical physicists or mathematicians [1,5-8] investigating its unique topological features, but also for an increasing number of architects and artists who consider the zero-volume bottle an authentic topological marvel and a source of endless creative inspiration. For those willing to understand the mechanisms that transform a simple cylinder into the popular "classical inverted sock" Klein bottle, a helpful pictorial explanation can be found in [9].

KBs are non-orientable surfaces, with a cross-cap number of 2 and an edge number of 0 . They can be easily constructed from Möbius bands (for simplicity, only half-twisted Möbius joints will be considered herewith), connecting the remaining open edges in a "parallel" manner, i.e., performing the same operation involved in transforming a rectangle into a cylinder until the structure is completely glued to a one-sided surface. It is well known that both tori and KBs, having Euler characteristics equal to 0 , may be covered with hexagonal tiles only and without the need for pentagons. They represent, for this reason, relevant examples of polyhexes, often referred to in the literature as toroidal and Klein bottle fullerenes. In this graphene era, our preference in this article is to name these surfaces paved with only hexagonal six-rings, graphenic nano-structures. The creation of polygonal faces with a number of edges other than six may be achieved using Stone-Wales (SW) rotations on the surface of these polyhexes to generate pentagon-heptagon pairs or other $r$-rings with $r \neq 6$ by simple iterations of multiple SW rearrangements. Various isomeric SW mechanisms to create and propagate innumerous kinds of topological defects in graphenic toroidal lattices have appeared in recent literature [10-14], and it is important to mention that these are all applicable to the case of graphenic KBs.

Furthermore, these nano-topo structures are related because the nano-surface's dynamics respect the graphenic projective plan; as for any nano-variety dynamics, they may be also related with quantum evolution, inherently at the nano-level. In this respect, although having as the common quantum nature the nano-confinement structure of space, the molecular topology (more precisely, chemical graph theory) and nano-architectures in general are sparsely connected with quantum information (e.g., $[15,16])$. As such, the classic quote of the quantum physicist Richard Feynman, that "there's plenty of room [i.e., enough space] at the bottom", has remained largely unexplored from the perspective of a quantum mechanical interpretation. Paradoxically, the Klein bottle structure was identified as having mathematical links with toroid systems, at least at their 3D immersing level, and with the quantum mechanical paradigm through the quaternion $4 \mathrm{D}$ representation, but not in a straight quantum (relativistic) dynamical interpretation, with the exception of some inputs in the quantum frontier-related literature (see the Appendix A). The present topographic endeavor may provide further insights into such quantum dynamics in the context of the significant interest at present in the potential future development of a unified science of nano-space shapes with consequences for mathematics (unitary manifold space variation), physics (quantum and relativistic unification by space shape dynamics), and chemistry (unification of chemical bond and bonding in extended nano-carbon spaces).

Methodologically, in the next section, we provide details about the construction of toroidal and Klein bottle fullerenes made with given numbers of hexagons $h$, atoms $N$, and bonds $B$. An introduction to topological distance-based descriptors (also including detailed calculations for small polyhexes) is also provided, and the general results derived for both graphs for large $N$ are presented. Topological similarities between toroidal and Klein bottle graphenic nano-systems are discussed, and the article concludes with preliminary indications about the relative chemical stability of these hexagonal systems. The graphenic $\mathrm{G}_{x, y}$ nano-structures considered here have a zigzag Möbius edge parallel to $x$ with dangling bonds closed across the direction $y$. The results and their topo-quantum dynamical interpretation are provided. 


\section{Method: Topological Invariants for Polyhex Graphs}

This section starts with a description of the method used to build the chemical graphs of the two graphenic nanostructures, tori and KBs. Then, distance-based topological invariants, such as the Wiener numbers $W$ and topological roundness $\rho$, are computed for some of these graphs.

In the current paper, we do not use the distorted parallelogram mesh often seen in the literature to build the polyhex graphs; instead, we adopt the quadrangular honeycomb mesh. The graphs shown in Figures $2-4$ are constructed by the usual $[12,13,17]$ C-shaped unit cell made by four atoms-1, 2, 3 , and 4-and which is translated $L_{M}$ times along $x$ and $L_{C}$ times in y, where the integers $L_{M}$ and $L_{C}$ indicate, respectively, the lengths of the Möbius-like edge (subject to the antiparallel sewing) and the cylindrical edge (parallel sewing), both expressed as the number of hexagons. The graphenic open lattice $G_{L_{M}, L_{C}}$ is made with $L_{C}$ rows with $L_{M}$ hexagons. The number $h$ of total hexagons in the $L_{C}$ belts is then $h=L_{M} \times L_{C}$. By closing the edges, the numbers of atoms $N$ and bonds $B$ in the graph are: $N=4 h, B=\frac{3 N}{2}=6 h$.
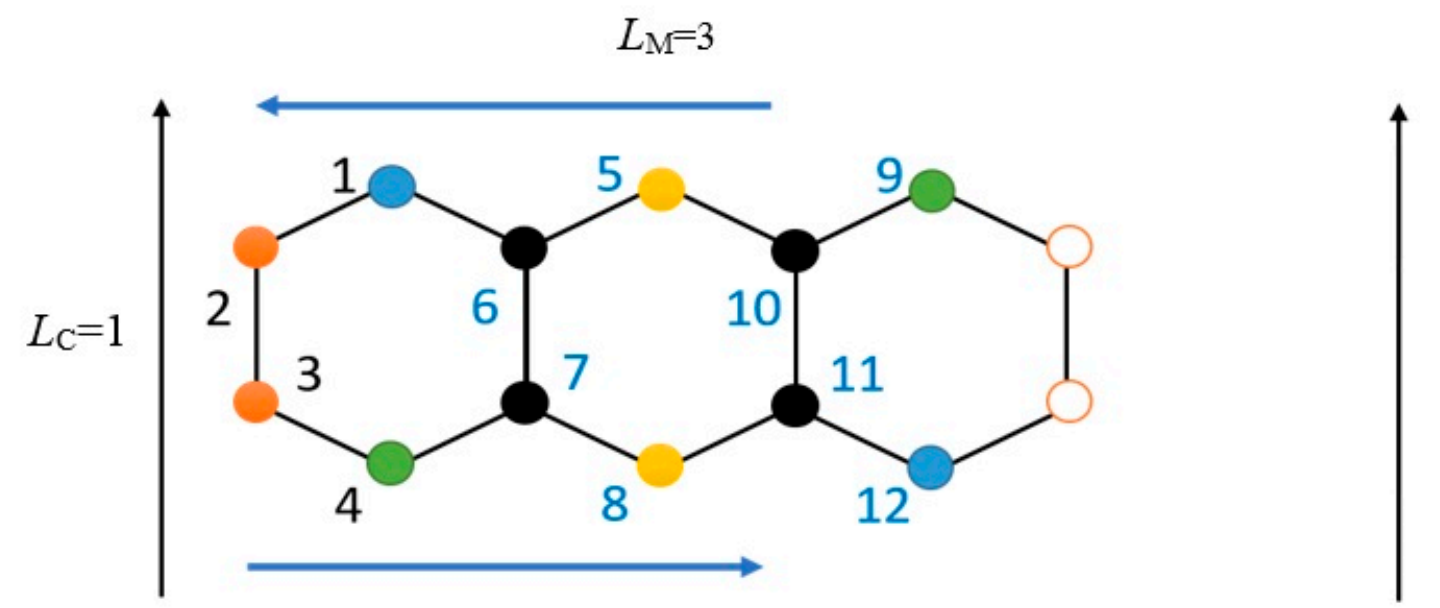

Figure 2. Polyhex lattice with $L_{M}=3, L_{C}=1$. The unit cell is the usual C-shaped cell consisting of the four atoms: $1,2,3$, and 4 . This graphenic lattice $G_{3,1}$ has open bonds along $y$ that are closed in a parallel manner along $x$ forming a cylinder: node 2 bounds 9 , and 3 bounds 12 . The graphenic torus $T_{3,1}$ has the open bonds along $x$ closed in a parallel manner along y: 1-4, 5-8, 9-12. By sewing the open bonds along $x$ in the antiparallel manner $(1-12,5-8,4-9)$, the Klein bottle $K B_{3,1}$ is built. Nodes 1,4 , $6,7,9,10,11$, and 12 are topologically equivalent to $\left\{b_{k}\right\}=\{3,5,3\}$, with shrunk eccentricity $\underline{\varepsilon}=3$; the remaining vertices $2,3,5$, and 8 share the coordination numbers $\left\{b_{k}\right\}=\{3,4,3,1\}$ and eccentricity $\bar{\varepsilon}=4$ of the $\mathrm{T}_{3,1}$ nodes.

The hexagon-tiled graphenic plane shows two sides and one edge. By changing the boundary conditions, the torus $\mathrm{T}_{L_{M}, L_{C}}$ and the Klein bottle $\mathrm{KB}_{L_{M}, L_{C}}$ have, respectively, numbers of sides (edges) equal to $2(0)$ and $1(0)$.

Here we present some practical examples. Figure 2 shows the $L_{M}=3, L_{C}=1$ honeycomb network. This graphenic lattice may be closed in two distinct topological manners, forming the torus $T_{3,1}$ or the Klein bottle $K B_{3,1}$ where $h=3, N=12$, and $B=18$. In both cases, the $L_{C}$ hexagons are closed in parallel along $x$. On the other edge, the dangling bonds belonging to the $L_{M}$ hexagons are closed in parallel or antiparallel along $y$ to form the $T_{3,1}$ torus or the $K B_{3,1}$ Klein bottle. Figure 3 shows more detail of how the two closed structures $T_{3,1}$ and $K B_{3,1}$ are built. The populations over the coordination shells change in a significant way depending on which of the two structures is considered. 


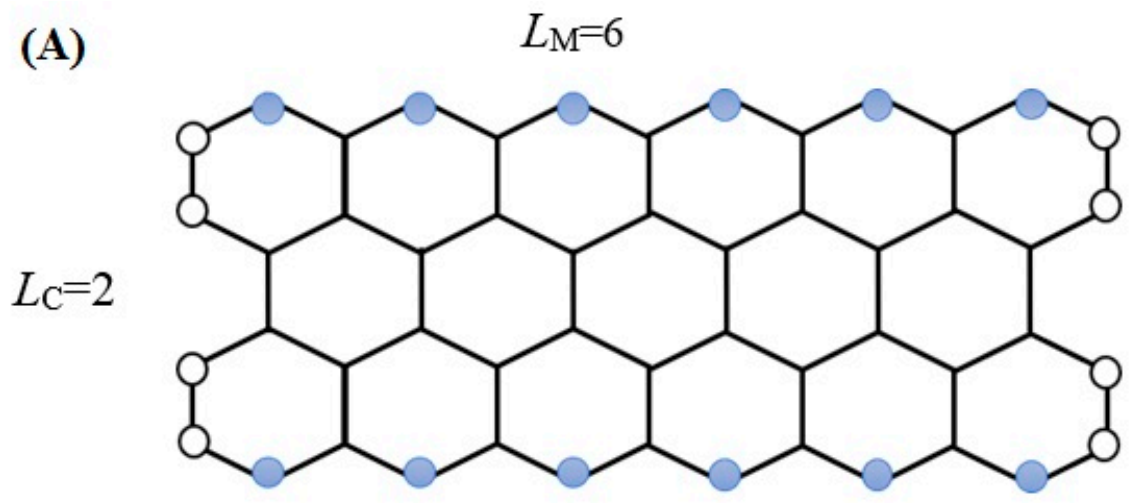

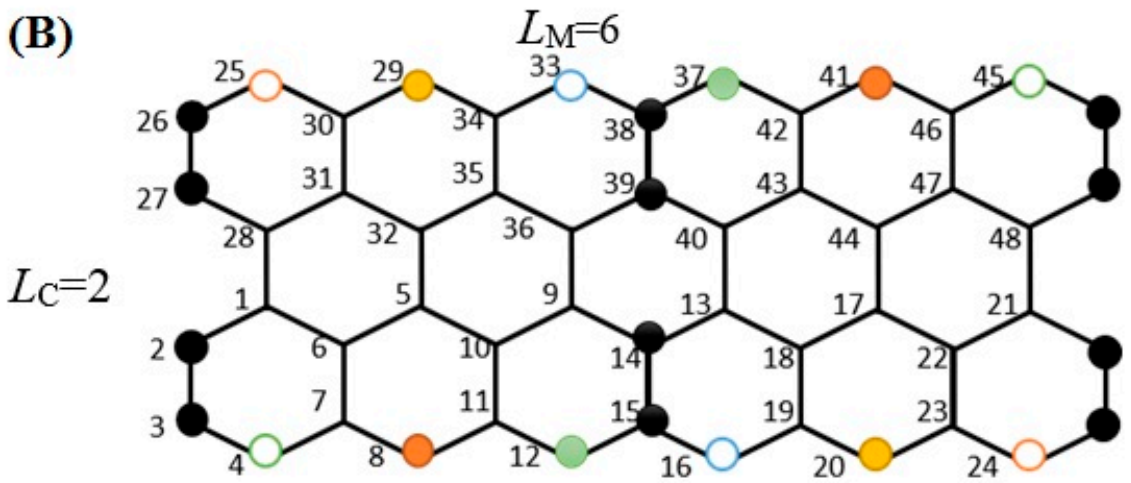

Figure 3. Polyhex lattices with $L_{M} 6, L_{C}=2$. (A) The toroidal fullerene $T_{(6,2)}$ is made of $N=48$ equivalent nodes with $\bar{w}=98$. Edges are closed to form the torus by sewing the balls with the same color in a parallel manner; (B) the Klein bottle $K B_{(6,2)}$ built by gluing, in an antiparallel manner, the nodes of the zigzag edge, such as 25-24, 29-20, ..., 45-4. Black circles have the same transmission of the torus' vertices, whereas the remaining circles have lower shrunken values, making $K B_{(6,2)}$ topologically more compact than $T_{(6,2)}$.

Indicating with $b_{i k}$ the number of $k$ - neighbors of the vertex $i$ in the graph, a quick calculation shows that all nodes in $T_{3,1}$ have the same set $\left\{b_{i k}\right\}=\{3,4,3,1\}$, with one node $j$ in the fourth coordination shell at the maximum distance $d_{i j}=4$. The maximum distance from $i$ determines the node eccentricity $\varepsilon_{i}=4$. The Klein bottle $K B_{3,1}$ shows some "toroidal" nodes with the same $\left\{b_{i k}\right\}$ set (namely the vertices $2,3,5$, and 8), and the remaining nodes have a reduced eccentricity $\varepsilon_{i}=3$ and $\left\{b_{i k}\right\}=\{3,5,3\}$. For example, vertex 1 in Figure 2 has three neighbors - 4, 8, and 10-in the third coordination shell but zero nodes in the fourth shell. 


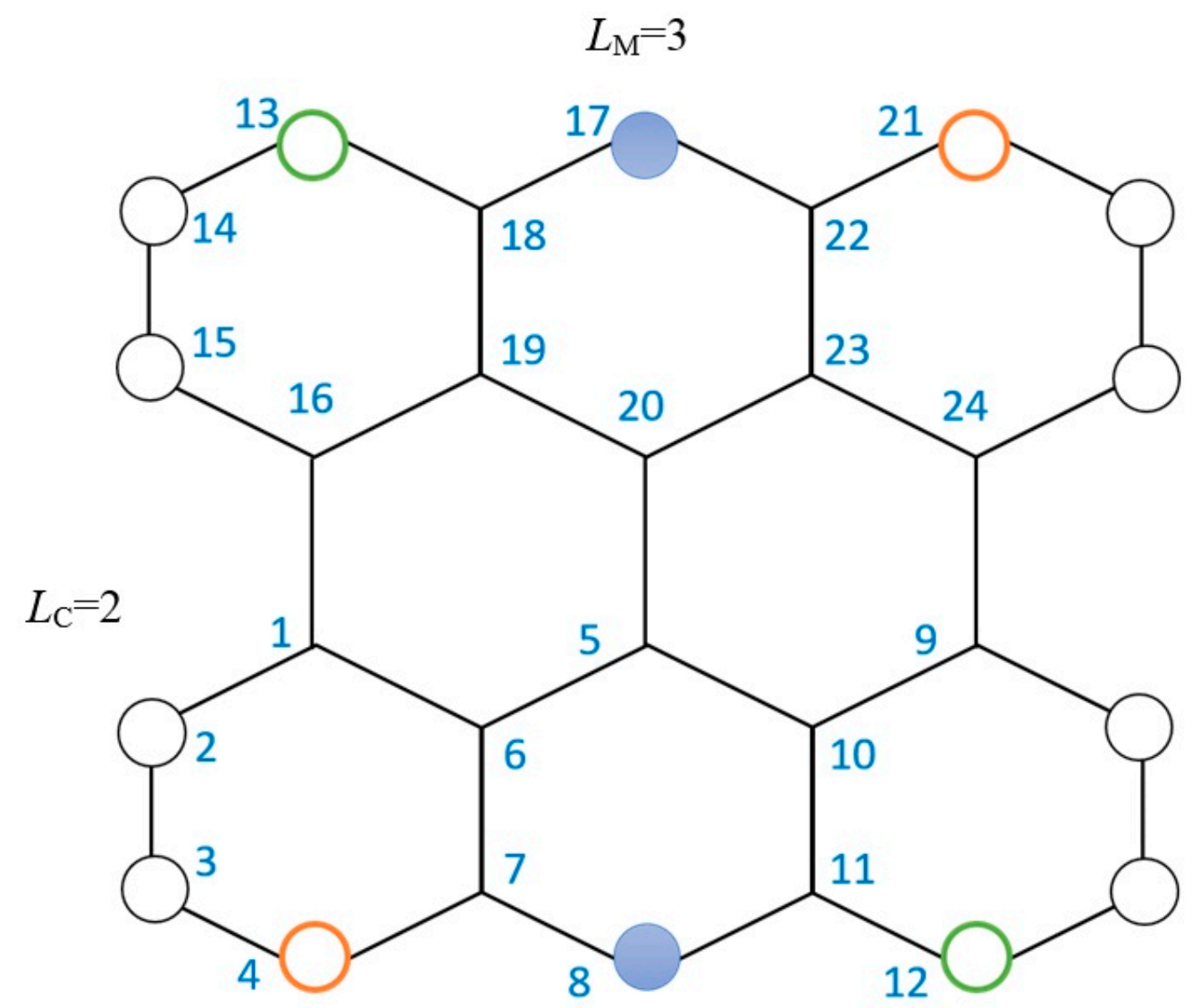

Figure 4. $\mathrm{KB}_{3,2}$ graph with $L_{M}=3, L_{C}=2$. Open bonds along $\mathrm{x}$ are sewn in the antiparallel manner (13-12, 17-8, 21-4); open bonds along y are close, in parallel. This Klein bottle is formed by $\mathrm{N}=24$ equivalent nodes with $\left\{b_{k}\right\}=\{3,6,8,5,1\}$, the same set shown by all nodes of the $T_{3,2}$ torus.

This effect of shortening the maximum eccentricity $M=\max \left\{\varepsilon_{i}\right\}$ of the graph (also called the graph diameter) is the topological signature of the antiparallel sewing acting on the dangling bonds placed along the zigzag Möbius edge. The transmission $w_{i}$ is defined in such a way:

$$
w_{i}=\frac{1}{2} \sum_{k=1}^{M} k b_{i k}
$$

and contributes to the Wiener index sum:

$$
W=\sum_{i=1}^{N} w_{i}
$$

The invariant $W$ represents a topological measure of the overall compactness of the graphenic structures. One can easily calculate $W\left(T_{3,1}\right)=144$ and $W\left(K B_{3,1}\right)=136$. Therefore, the $(3,1)$ Klein bottle results in a more compact structure if compared with the nano-torus with equal edges. The topological invariant $\rho_{E}$, called extreme topological efficiency [18,19] or extreme topological roundness [20], is defined as the ratio between the extreme value in the transmission set of a given graph:

$$
\rho_{E}=\frac{\max \left\{w_{i}\right\}}{\min \left\{w_{i}\right\}}
$$

The invariant of Equation (3) is able to select stable structures such that the cases of fullerenes $\mathrm{C}_{60}$ or $\mathrm{C}_{84}[21,22]$ tend to minimize the $\rho_{E}$ topological descriptor. Tori $\mathrm{T}_{L_{M}, L_{C}}$ have $\rho_{E}=1$, which is different from the situation for the Klein bottles. In our example, $\rho_{E}\left(K B_{3,1}\right)=\frac{12}{11}>\rho_{E}\left(T_{3,1}\right)$, evidencing the greater topological stability of the torus. 
Our topological modeling approach is based on the minimization of the topological invariants promoting the most compact (minimizing $W$ values) and most round (minimizing $\rho_{E}$ values) structures as the most stable systems among a given set of isomers. This paper studies the effects of the topology in influencing the relative stability of the two isomeric structures, tori and Klein bottles, based on the same graphenic plane $\mathrm{G}_{L_{M}, L_{C}}$, in particular, the variation of the $\left\{b_{i k}\right\}$ sets following the different closures of the zigzag Möbius edge. We conclude the article by reporting an original size-dependence effect that, for certain circumstances, makes tori and KBs topologically indistinguishable.

Table 1 lists the values of the invariants for the cases in which the length of the zigzag Möbius edge triples the other edge $L_{M}$ and $L_{C}=3 L_{M}$. This is an evidently arbitrary choice intended only to represent all cases with $L_{C}<L_{M}-1$.

Table 1. Topological classes for the vertices of the graph $K B_{6,2}$, including eccentricity $\epsilon_{i}$, transmission $w_{i},\left\{b_{i k}\right\}$ sets, Wiener number $\mathrm{W}$, and extreme topological efficiency $\rho_{E}$; multiplicity is given in brackets. $T_{6,2}$ descriptors hold for all nodes of the toroidal polyhex and coincide with the last class of $K B_{6,2}$.

\begin{tabular}{|c|c|c|c|}
\hline \multicolumn{4}{|l|}{$K B_{6,2} \mathrm{~W}=4504 ; \rho_{E}=98 / 91=1.0769$} \\
\hline$V$ & $\left\{b_{i k}\right\}$ & $\epsilon_{i}$ & $w_{i}$ \\
\hline (8) v5 v8 v17 v20v29 v32 v41 v44 & 369121151 & 7 & 91 \\
\hline (16) v6 v7 v10 v11 v18 v19 v22 v23 v30 v31 v34 v35 v42 v43 v46 v47 & 369111161 & 7 & 92 \\
\hline (16) v1 v4 v9 v12 v13 v16 v21 v24 v25 v28 v33 v36 v37 v40 v45 v48 & 36910973 & 7 & 95 \\
\hline (8) v2 v3 v14v15v26 v27 v38v39 & 36998741 & 8 & 98 \\
\hline \multicolumn{4}{|l|}{$\mathrm{T}_{6,2} \mathrm{~W}=4704 ; \rho_{E}=1$} \\
\hline (48) $v 1, v 2, \ldots, v 47, v 48$ & 36998741 & 8 & 98 \\
\hline
\end{tabular}

Figure 3 illustrates the topological analysis of the two graphs $T_{3 l, l}$ and $K B_{3 l, l}$ with $l=2, N=48$. All $N$ nodes of the torus show the same transmission (1) value $\bar{w}=98$ with $M=8$. The interesting (and expected) fact is that eccentricities of most of the nodes of $K B_{6,2}$ are smaller than the graph diameter $M$. This reduction of the eccentricity is a clear effect induced by the Möbius-like sewing of the zigzag edge. The $K B_{6,2}$ diameter is still $M=8$, but this value only concerns the few nodes circled in black in Figure $3 b$. All the remaining nodes on the surface of the KB have eccentricities equal to $M-1$, suggesting the existence of a general topological effect that we call eccentricity shrinkage, Equation (4b). Eccentricity shrinkage is a topological phenomenon that has been previously reported [23] for Möbius $1 \mathrm{D}$ graphenic (open) strips and has the overall topological effect of making $\mathrm{KB}_{L_{M}, L_{C}}$ topologically more compact then $\mathrm{T}_{L_{M}, L_{C}}$ when $L_{C}<L_{M}-1$. For the polyhexes in Figure 3 , the Wiener index of Equation (2) computed values $W(K B)=4504$ and $W(T)=4704$ confirm that $K B_{6,2}$ possesses an augmented compactness over $T_{6,2}$, that is, $W(K B)<W(T)$. It is worth noting that $W(T)=N \bar{w}$. The Klein bottle fullerene $K B_{6,2}$ has a broader range of topological transmission values, from $\underline{w}=91$ to $\bar{w}=98$. Table 1 details the list of topological descriptors for each node of the $K B_{6,2}$ graph, including eccentricity and $\left\{b_{i k}\right\}$ sets; descriptors for $T_{6,2}$ are also provided for completeness (vertices are labeled as shown in Figure 3). Topological descriptors group the $N=48$ nodes of the $\mathrm{KB}_{6,2}$ graph into four classes of topological equivalent vertices, with the multiplicity given in brackets in the first column of Table 1 . The ${ }^{13} \mathrm{C}$ NMR resonance spectrum of the carbon $\mathrm{C}_{48}$ nanostructure has such a Klein bottle topology made of four peaks with relative intensities $1,1,2$, and 2 . We end these considerations with Table 2, which shows the eccentricity shrinkage in passing from $T_{3 l, l}$ to $K B_{3 l, l}$ graphs for $l=1,2, \ldots, 9,10$. 
Table 2. For the family of graphs $K B_{3 l, l}$, the eccentricity $\left\{\epsilon_{i}\right\}$ values are compared to the tori $T_{3 l, l}$ eccentricity $\bar{\varepsilon}$ for $l=1,2, . ., 10$. Indicating with $\underline{\varepsilon}=\min \left\{\epsilon_{i}\right\}$, the topological shrinkage $\underline{\varepsilon}<\bar{\varepsilon}$ of the Klein bottles is a peculiar outcome of the current study.

\begin{tabular}{|c|c|c|}
\hline$l$ & $K B_{3 l, l}\left\{\epsilon_{i}\right\} \underline{\varepsilon}=\min \left\{\epsilon_{i}\right\}$ & $T_{3 l, l} \bar{\varepsilon}$ \\
\hline 1 & $3,4 \underline{\varepsilon}=3$ & 4 \\
\hline 2 & $7,8 \bar{\varepsilon}=7$ & 8 \\
\hline 3 & $10,11,12 \underline{\varepsilon}=10$ & 12 \\
\hline 4 & $13,14,15,1 \overline{6} \underline{\varepsilon}=13$ & 16 \\
\hline 5 & $17,18,19,20 \underline{\varepsilon}=17$ & 20 \\
\hline 6 & $20,21,22,23,2 \overline{4} \underline{\varepsilon}=20$ & 24 \\
\hline 7 & $23,24,25,26,27,28 \underline{\varepsilon}=23$ & 28 \\
\hline 8 & $27,28,29,30,31,32 \underline{\bar{\varepsilon}}=27$ & 32 \\
\hline 9 & $30,31,32,33,34,35,3 \overline{6} \underline{\varepsilon}=30$ & 36 \\
\hline 10 & $33,34,35,36,37,38,39,4 \overline{0} \underline{\varepsilon}=33$ & 40 \\
\hline
\end{tabular}

With the examples above, we showed the existence of the general mechanism of the topological effect we have called eccentricity shrinkage, which is a peculiar outcome of the current study; see Equation (4b).

This mechanism implies that, by indicating with $\underline{\varepsilon}$ the minimum value of the eccentricities of the Klein bottle $K B_{L_{M}, L_{C}}$ graph and with the symbol $\bar{\varepsilon}$ the eccentricity of the $T_{L_{M}, L_{C}}$ torus with the same pair of edges $L_{M}, L_{C}$, one can find:

$$
\underline{\varepsilon} \leq \bar{\varepsilon}
$$

When the eccentricity shrinkage mechanism holds, the inequality is strictly respected:

$$
\underline{\varepsilon}<\bar{\varepsilon}
$$

The main result of the present study is summarized in the following novel conjecture concerning toroidal and Klein bottle fullerenes $K B_{L_{M}, L_{C}}$ and $T_{L_{M}, L_{C}}$ :

Polyhex similarity conjecture. For a given integer value $L_{\mathrm{M}}$, the shrinkage of the topological eccentricity does not hold over the threshold $L_{C} \geq Ł_{C}$ with $\succeq_{C}=L_{M}-1$.

Formally, we then have:

$$
\varepsilon=\bar{\varepsilon}
$$

in the range:

$$
L_{C} \geq E_{C}
$$

where

$$
Ł_{C}=L_{M}-1
$$

Thus, for polyhexes $K B_{L_{M}, L_{C} \geq L_{C}}$ and $T_{L_{M}, L_{C} \geq L_{C}}$, the equality $\underline{\varepsilon}=\bar{\varepsilon}$ holds. By contrast, for $K B_{L_{M}, L_{C}<L_{C}}$ and $T_{L_{M}, L_{C}<L_{C}}$, the inequality $\underline{\varepsilon}<\bar{\varepsilon}$ holds strictly, making Klein bottle fullerenes topologically more compact than the toroidal ones.

The next section is devoted to the explanation of the above main result (5a), i.e., the existence of a critical size $L_{C}$ that makes toroidal and Klein bottle polyhexes topologically indistinguishable and vertex transitive.

\section{Results: Topological Similarities between Toroidal and Klein Bottle Polyhexes}

This section aims to illustrate the above polyhex similarity conjecture using examples extracted by the numerical investigations supporting it. We start by recalling the data reported in Table 1 for the two toroidal and Klein bottle polyhexes shown in Figure 3. The eccentricity $\bar{\varepsilon}=8$ of the torus $T_{6,2}$ is the upper limit of the $K B_{6,2}$ eccentricities $\left\{\varepsilon_{i}\right\}=\{7,8\}$ with the minimum value $\underline{\varepsilon}=7$. 
Figure 4 enables us to provide more computational details about the simple system $K B_{3,2}$, that is, a graph with $L_{\mathrm{M}}=3$, and $L_{\mathrm{C}}=2$ with $\biguplus_{\mathrm{C}}=2$, fully compatible with the conditions of Equation (5), leading to the topological similarity with the torus $T_{3,2}$. We start with the graphenic open lattice $G_{3,2}$ made by $N=24$ vertices, and we first close the $y$ edge in the usual cylindrical manner, with, for example, node 14 bonding vertices 13,15 , and 21 , and so on. We know from the Klein bottle $K B_{3,1}$ represented in Figure 2 that some nodes (such as vertex 4 ) show $\underline{\varepsilon}=3$ shrunk eccentricity with respect to the node of the torus $T_{3,1}$, having eccentricity $\bar{\varepsilon}=4$. In particular, vertex 4 in the Klein bottle has three vertices in the maximum distance coordination shell $d_{4,1}=d_{4,5}=d_{4,11}=3$. By contrast, in the torus $T_{3,1}$, the same vertex still has three nodes in the third coordination shell $d_{4,9}=d_{4,5}=d_{4,11}=3$ but also one node 10 at the maximum distance $d_{4,10}=4=\bar{\varepsilon}$. When the graphenic open lattice $G_{L_{M}, L_{C} \geq L_{C}}$ is built respecting the conditions of Equations $(5 b, 5 c)$ relating to the sizes of the two edges, the topological distances of the toroidal and Klein bottle polyhexes will change in such a way that all the nodes of both graphs show the same eccentricity $\underline{\bar{\varepsilon}}=\varepsilon$ and the same set of coordination numbers $\left\{b_{k}\right\}$ with $k=1,2, . ., \underline{\varepsilon}$. Klein bottle polyhexes $K B_{L_{M}, L_{C} \geq L_{C}}$ are made by the vertex transitive graph with the same invariants $(1,2,3)$ shown by the nodes of the $T_{L_{M}, L_{C} \geq L_{C}}$ tori.

The following numerical test focuses again on node 4 of the $T_{3,2}$ torus and $K B_{3,2}$ graphs in Figure 4. For the torus, the three nodes with distances equal to 4 are vertices 10,16,20,22, and 24, and one node is at distance $d_{4,23}=5=\bar{\varepsilon}$. In the Klein bottle $K B_{3,2}$, the antiparallel closure makes node 23 closer to node 4 , at $d_{4,23}=3$, with four nodes in the fourth coordination shell-10,16,18,20, and 24-and one node at the maximum distance $d_{4,19}=5$. This example fortifies the conjectured similarity among tori and $K B$ s when $L_{C} \geq Ł_{C}$.

Table 3 shows a larger numerical test confirming the main result of Equation (5a) of the current work and therefore supports the existence of a critical size $\rfloor_{C}=L_{M}-1$ of Equation (5c) making toroidal and Klein bottle polyhexes topologically indistinguishable. For the two families of graphs $\mathrm{KB}_{L_{M}, L_{C}}$, $L_{M}=5,6$ and $L_{C}=1,2, \ldots$; the eccentricity $\left\{\epsilon_{i}\right\}$ values are compared with the eccentricity $\bar{\varepsilon}$ of the isomeric tori. In both cases, the topological shrinkage of the eccentricity $\underline{\varepsilon}<\bar{\varepsilon}$ takes place in the region $L_{C}<t_{C}$, confirming the outcome of the current study. We also note that $\bar{\varepsilon}=\underline{\varepsilon}=2 L_{C}$ for $L_{C}>t_{C}$ in both cases when $L_{M}=5,6$.

The current results show that the Klein bottle $\mathrm{KB}_{L_{M}, L_{C}}$ cubic graphs are topologically indistinguishable from the toroidal lattices $T_{L_{M}, L_{C}}$ with the same size $\left(N, L_{C}, L_{M}\right)$ when $L_{C} \geq t_{C}$; see Equation (5c).

This result implies that an observer sitting on a KB node sees (i) the same coordination numbers $\left\{b_{k}\right\}$ that characterize the vertices of the isomeric torus $T_{L_{M}, L_{C}}$; (ii) that the Klein bottle graph has become vertex transitive with all nodes sharing the same $\left\{b_{k}\right\}$.

A graph traveler arriving at the nodes of the Klein bottle fullerenes is no longer able to distinguish among them nor to determine if they are staying on a torus by simply measuring the chemical distances. This size-induced symmetry transition for Klein bottle cubic graphs represents a relevant topological effect with deep influences on the electronic properties of these theoretical graphenic nanosystems. Such intriguing topological phenomena may relate to the formation and propagation of the quasi-quantum bosonic particle called the bondon [24], carrying certain topological chemical bonding information along a chemical structure-with more thermochemical observable effects when it relates to extended graphenic systems $[25,26]$. Bondonic theory may also eventually explain the homologies of chemical structures, as proven by the aid of the symmetry breaking mechanism, thus balancing the fermionic vs. bosonic (in)formation with direct influence on the aromatic or reactivity degree of a certain compound [27]. 
Table 3. For $\mathrm{L}_{\mathrm{M}}=5,6$, the eccentricities $\left\{\epsilon_{i}\right\}$ of the $\mathrm{KB}_{L_{M}, L_{C}}$ graphs are compared with the toroidal case, $\mathrm{L}_{\mathrm{C}}=1,2, \ldots$. Graphs with $L_{C}<L_{M}-1$ show the topological shrinkage of the eccentricity $\underline{\varepsilon}<\bar{\varepsilon}$ and Wiener index $\mathrm{W}_{\mathrm{KB}}<\mathrm{W}_{\mathrm{T}}$; for $L_{C} \geq L_{M}-1$, the $\mathrm{KB}$ graphs are topologically equivalent, $\underline{\varepsilon}=\bar{\varepsilon}$, to the tori $\mathrm{T}_{L_{M}, L_{C}}$ of the same sizes with $\mathrm{W}_{\mathrm{KB}}=\mathrm{W}_{\mathrm{T}}$.

\begin{tabular}{|c|c|c|c|c|}
\hline \multicolumn{5}{|c|}{$L_{M}=5, t_{C}=4 ;$ for $L_{C} \geq E_{C}$, the two polyhexes are equivalent. } \\
\hline$L_{C}$ & $\mathrm{~KB}_{5, L_{C}}\left\{\epsilon_{i}\right\} \varepsilon=\min \left\{\epsilon_{i}\right\}$ & $W_{K B}$ & $\mathrm{~T}_{5, L_{C}} \bar{\varepsilon}$ & $W_{T}$ \\
\hline 1 & $4,5,6 \underline{\varepsilon}=4$ & 528 & 6 & 600 \\
\hline 2 & $6,7 \underline{\varepsilon}=6$ & 2800 & 7 & 2880 \\
\hline 3 & $7,8 \underline{\varepsilon}=7$ & 7632 & 8 & 7680 \\
\hline 4 & $9 \underline{\varepsilon}=9$ & 16,000 & 9 & 16,000 \\
\hline 5 & $10 \underline{\bar{\varepsilon}}=10$ & 29,000 & 10 & 29,000 \\
\hline 6 & $12 \underline{\bar{\varepsilon}}=12$ & 48,000 & 12 & 48,000 \\
\hline 7 & $14 \underline{\bar{\varepsilon}}=14$ & 74,200 & 14 & 74,200 \\
\hline 8 & $16 \underline{\bar{\varepsilon}}=16$ & 108,800 & 16 & 108,800 \\
\hline 9 & $18 \bar{\varepsilon}=18$ & 153,000 & 18 & 153,000 \\
\hline 10 & $20 \underline{\varepsilon}=20$ & 208,000 & 20 & 208,000 \\
\hline 15 & $30 \overline{\bar{\varepsilon}}=30$ & 687,000 & 30 & 687,000 \\
\hline 20 & $40 \underline{\bar{\varepsilon}}=40$ & $1,616,000$ & 40 & $1,616,000$ \\
\hline 25 & $50 \underline{\bar{\varepsilon}}=50$ & $3,145,000$ & 50 & $3,145,000$ \\
\hline \multicolumn{5}{|c|}{$L_{M}=6, \iota_{C}=5$; for $L_{C} \geq E_{C}$, the two polyhexes are equivalent. } \\
\hline$L_{C}$ & $\mathrm{~KB}_{6, L_{C}}\left\{\epsilon_{i}\right\} \varepsilon=\min \left\{\epsilon_{i}\right\}$ & $W_{K B}$ & $\mathrm{~T}_{6, L_{C} \bar{\varepsilon}}$ & $W_{T}$ \\
\hline 1 & $4,5,6,7 \underline{\varepsilon}=4$ & 860 & 7 & 1008 \\
\hline 2 & $7,8 \varepsilon=7$ & 4504 & 8 & 4704 \\
\hline 3 & $8,9 \underline{\varepsilon}=8$ & 12,084 & 9 & 12,240 \\
\hline 4 & $9,10 \bar{\varepsilon}=9$ & 24,880 & 10 & 24,960 \\
\hline 5 & $11 \underline{\varepsilon}=11$ & 44,400 & 11 & 44,400 \\
\hline 6 & $12 \underline{\bar{\varepsilon}}=12$ & 72,288 & 12 & 72,288 \\
\hline 7 & $14 \underline{\bar{\varepsilon}}=14$ & 110,544 & 14 & 110,544 \\
\hline 8 & $16 \bar{\varepsilon}=16$ & 160,896 & 16 & 160,896 \\
\hline 9 & $18 \underline{\bar{\varepsilon}}=18$ & 225,072 & 18 & 225,072 \\
\hline 10 & $20 \underline{\bar{\varepsilon}}=20$ & 304,800 & 20 & 304,800 \\
\hline 15 & $30 \overline{\bar{\varepsilon}}=30$ & 997,200 & 30 & 997,200 \\
\hline 20 & $40 \underline{\bar{\varepsilon}}=40$ & $2,337,600$ & 40 & $2,337,600$ \\
\hline 25 & $50 \underline{\bar{\varepsilon}}=50$ & $4,542,000$ & 50 & $4,542,000$ \\
\hline
\end{tabular}

We end this section with some preliminary considerations concerning the chemical stability of a graphenic structure having the Klein bottle topology. Pioneering work on this subject may be found in a previous study [28]. Here, we report two concurrent topological characteristics that make the Klein bottle $\mathrm{KB}_{L_{M}, L_{C}}$ a good candidate for being a stable and synthesizable carbon nanostructure.

The above statement is based on the two main outcomes of the present study:

$>L_{C}<Ł_{C}$ : in this region, the eccentricity shrinkage of Equation $(4 b)$ holds and the Klein bottle is therefore more compact than the toroidal graphenic structure with equal size. The Wiener indices (2) of the Klein bottles are lower than those of the tori; see Table 3.

$>L_{C} \geq t_{C}$ : in this region, the conjectured topological similarity of Equation (5a) holds and the Klein bottle thus becomes topologically equivalent to the isomeric toroidal polyhex sharing the same values of $W$ and $\rho_{E}$; see Figure 5 . 


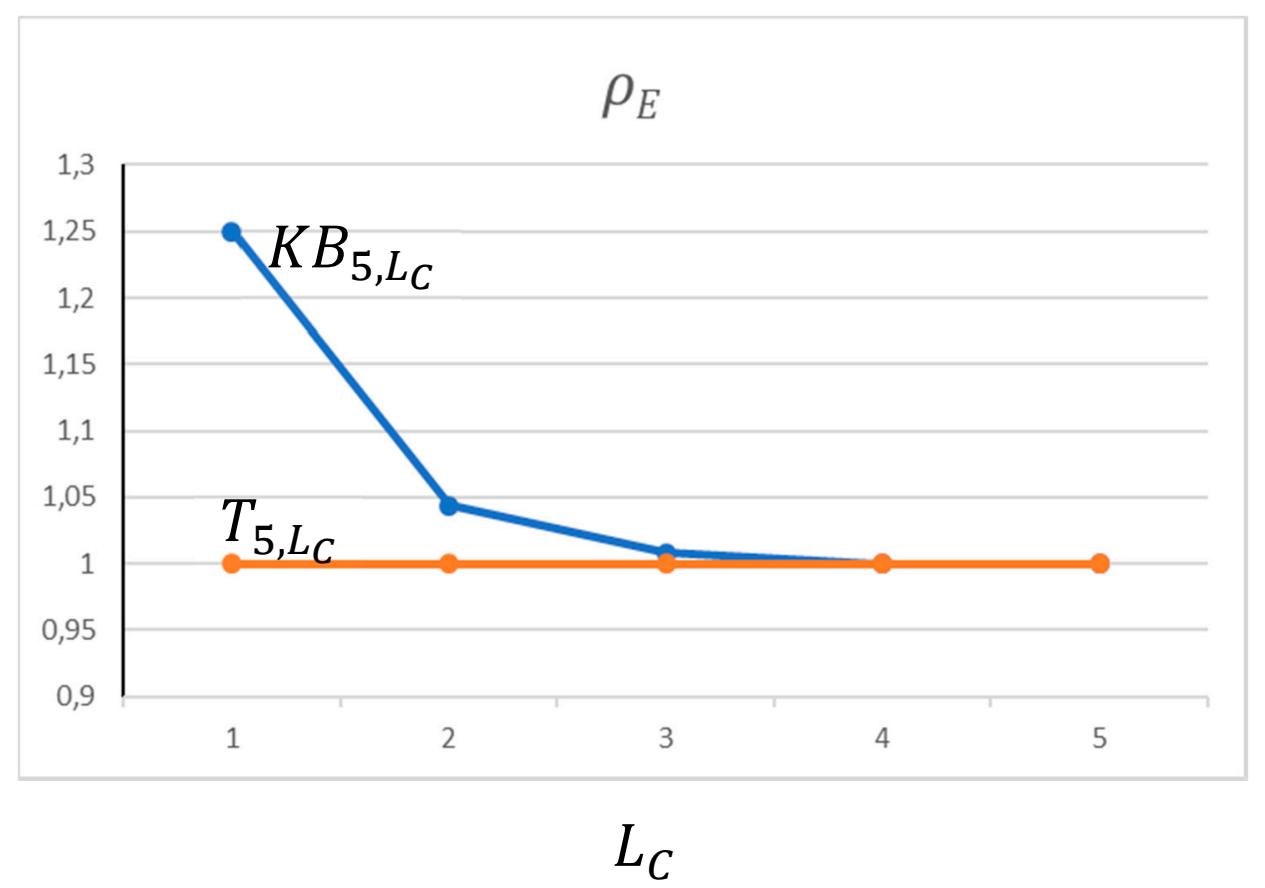

Figure 5. For $L_{M}=5, t_{C}=4$, and $L_{C}=1,2, \ldots, 5$, the topological roundness $\rho_{E}$ is plotted for both $\mathrm{KB}_{L_{M}, L_{C}}$ and $\mathrm{T}_{L_{M}, L_{C}}$ isomeric graphs; when $L_{C} \geq E_{C}$, the Klein bottle graphs are topologically equivalent to the tori $\mathrm{T}_{L_{M}, L_{C}}$.

The graphenic tori are a useful representation of the graphene plane with periodic boundary conditions imposed along the directions of both $x$ and $y$ of the honeycomb plane. Graphene has been recognized for the past 16 years as a stable carbon allotrope [29]. According to the present findings, both nano-structures show, for $L_{C} \geq E_{C}$, the same values of graph compactness and roundness (i.e., similarity conjecture), which are two key topological properties that largely influence the stability of the systems [21,22].

This fact suggests the possibility that Klein bottle systems can be formed because of their topological stability, which is comparable to that of the toroidal polyhexes. In the future, more detailed investigations will be devoted to determining under which conditions the Klein bottle fullerenes will be able to be produced in nature or in laboratories.

Remarkably, the present findings related to the conditions of the topological equivalence of the Klein bottle and torus may suggest another potentially useful interpretation of quantum mechanics at present, particularly when related to the space-time structural dynamics. The present endeavor offers a workable graphene-related nano-topological argument in favor of Everett's theory of many worlds [30-32]. Precisely, it suggests a certain degree of complexification, i.e., over a critical size $t_{C}=L_{M}-1$ of Equation (5c), the toroidal and Klein bottle polyhexes are topologically indistinguishable. Thus, they coexist, being superimposed or topologically entangled, in terms of quantum information theory. Accordingly, the two graphenic nano-systems may be quantum interrelated by the inter-correlated (Klein bottle, KB, and tori, T) wave functions, for example, by the entangled wave functions' symmetry transition:

$$
\int \Psi_{K B}(\{x\}) \Psi_{T}\left(\left\{y-\lambda_{T} E_{K B} t_{+}\right\}\right) d \tau=\int \Psi_{K B}\left(\left\{x-\lambda_{K B} E_{T} t_{-}\right\}\right) \Psi_{T}(\{y\}) d \tau
$$

In Equation (6), the two topological structures mutually measure each other in an entangled state with energies $E_{K B}$ and $E_{T}$ as the forward and reversal times of $t_{+}$and $t_{-}$flows, and with the respective ordering (i.e., observably related) of parameters of $\lambda_{T}$ and $\lambda_{K B}$. The future challenge will be to properly assess the topological parameters of the manifold observable many-world physical factors 
(e.g., establishing the degree to which the nano-structural and topological critical extended parameter $L_{C}$ accounts for the forward and reversal time, in a topo-quantum space-time unification approach, or the degree to which the topological roundness $\rho_{E}$ influences the observable (e.g., thermochemical, nuclear, magnetic, and electronic) parameters through the order parameters $\lambda_{K B, T}$, and so on).

Moreover, with the further exploration of other topological manifolds that are derived or transformed from the graphenic projective surface, one will eventually establish the graphenic branching-states as the true dynamic quantum space in which the entire nano-world coexists at "the bottom of the world", according to the famous terms of Richard Feynman's prediction, yet with the "curvature/potential" emerging into the observable and peculiarly controlled nano-chemical synthesizable structures.

\section{Conclusions}

By varying the topology of the polyhexes, from tori to Klein bottles, we observed the general mechanism that we called the eccentricity shrinkage consisting of the relationship $\underline{\varepsilon}<\bar{\varepsilon}$ between the eccentricities of tori $\bar{\varepsilon}$ and Klein bottles $\underline{\varepsilon}$. Moreover, a newly conjectured topological symmetry between the two kinds of graphs occurs when the critical size $L_{C} \geq t_{C}$ is imposed on the systems. In particular, under this condition, the translation invariance typical of the graphenic tori also becomes a topological property of the Klein bottle polyhexes, thus making these Klein bottle graphs vertex transitive, and suggesting that they may have the same topological stability of graphene. Therefore, they may be synthesizable as actual chemical structures. This is an intriguing possibility that is worthy of further theoretical and experimental (quantum) investigation.

Furthermore, as recently launched, Wolfram's computational unification of curved space with quantum theory with the aid of topological rolled and curved surfaces and particles (i.e., wave-packaging; equivalence of space curvature; and the topologically allied indices of torsion, time-space curvature, acceleration, bifurcations, topological evolving manifolds, many-worlds superposition and interference, and entanglement) offers a mathematically and computationally wider framework for the present topological Klein bottle/tori equivalence approach [33]. As such, the so-called (as abstracted from Wolfram's writings) branchial space, branchial motion, entanglement horizon, or maximal speed of motion (that is, $\zeta$, with respect to the maximum topological eccentricity of a given graphenic nano-space) in branchial space is eventually related to the quantum Zeno effect. A generalization of Einstein's space curvature invariance $\rho$ (with respect to the sphericity index of a given graphenic nano-space) occurs in conjunction with the multiway causal graph or the space-time curvature relating to the uncertainty quantum principle. All such tools for the geometrization of the complexity space (of the cosmos, nature, and nano-materials) may ultimately result in the nano-topo unification of the quantum and relativity theories (that is, both special and general) through the manifolds of many-world (viz. quantum), space-time coupled (or entangled) dynamics (see Appendix A). It is nevertheless true that nano-topologies in general and those specifically based on or derived from a graphenic nano-projective space may offer a computational experimental space for simulating and understanding (at the ontological level) the micro-folded and macro-unfolded universes. Such endeavors should continue to be necessary in the coming years.

Author Contributions: M.V.P. and O.O. established the conceptual framework, discussion, and conclusions, and assembled the paper; O.O. performed the calculations; and M.V.P. conducted the literature screening and contributed the quantum-relativity topological interpretation. All authors have read and agreed to the published version of the manuscript.

Funding: Mihai V. Putz acknowledges his contribution to this work within the Nucleus-Programme under the project PN-19-22-01-02 and its 2020 renewal as funded by the Romanian Ministry of Education and Research. Ottorino Ori is a permanent fellow of the Laboratory of Structural and Computational Physical-Chemistry for Nanosciences and QSAR, Biology-Chemistry Department, Faculty of Chemistry, Biology, Geography, West University of Timisoara, as well as of the Laboratory of Renewable Energies-Photovoltaics, R\&D National Institute for Electrochemistry and Condensed Matter, Timișoara, Romania.

Conflicts of Interest: The authors declare no conflict of interest. 


\section{Appendix A. On Topological and Quantum Coverings of Nano-Space}

The coverage of nano-space by carbon allotropes has been a constant source of fascination, in terms of both topological and chemical synthesis. During the past 50 years, significant milestones have been represented by the discoveries (via experimental evidence and structural characterization) of fullerene [34] and graphene [35]. Accordingly, novel nano-architecture spaces have emerged in single-walled nanotubes (SWNTs) [36]. The coalescence reactions of SWNTs [37] are considered classical examples of nano-chemistry. Furthermore, from an exotic perspective, foam-like carbon structures related to schwarzite [38] represent infinite periodic minimal surfaces of negative curvature containing polygons of size larger than hexagons (in comparison to graphite) that induce a negative curvature. Units of such structures appear in nanotube junctions, produced in an electron beam [39], with wide potential applications in molecular electronics [40]. In addition, although carbon nano-chemistry has resulted in significant technological and experimental advances, a wide range of challenges remain to be conquered. However, nano-carbon allotropes have revealed experimental surfaces that allow unique and innovative connection with mathematical and topological experiments [41,42] - that is, employing the mathematical laboratory in the analysis of examples, testing of ideas, or search for new patterns. In this context, a plethora of theoretical models have been advanced either for already existing nanostructures (as outlined previously) or experimentally designed new nanostructures, in a mathematical-topological manner. For instance, the first fullerene computational modeling was undertaken nearly three decades ago by topological means (i.e., chemical graphs) $[43,44]$ and subsequently advanced to renewed means [45]. These have been accompanied by systematic studies of the embedding of the hexagonal trivalent $(6,3)$ net; that is, the drawing of a graph on a (closed) surface with no crossed lines (cf., the Schäfli notation). In the torus or cylinder, this is predominantly achieved by the well-known method of graphite zone-folding; see Figure A1, top [46-49]. More recent developments include density and functional theory-based investigations [50], and studies of the negative curvature of nano-architectures [51,52]. These latter developments provide an explanation for natural micro-pores with low densities appearing in natural materials as zeolites. In particular, such pores can be simulated, e.g., by structures tessellated entirely by heptagons (e.g., the Klein tessellation) embedded into infinite periodic surfaces of negative curvature of genus $>1$. At this point, one arrives at the so-called Smale's paradox of topology, which represents, in dynamical space, spheres and tori everted (i.e., turned inside out) by smooth transformations. The paradox expresses that fact that, although the direct surface is an orientable one, the everted one means that the act of turning the surface inside-out implies that the half-way surface is non-orientable in any instance. An object that originates from such an operation is the Klein bottle (Figure A1, bottom) [53].

In addition to having no direct $3 \mathrm{D}$ realization, this appears in the $3 \mathrm{D}$ embedded forms of Figure A1 with a 2D surface carrying neither a global surface outside nor a global surface inside. Moreover, it transcends the ordinary topological half-way surface transformation, with no such transformation occurring in the case of the Klein bottle, which is the only remaining case related to holonic transformation. At this point, the topology should be linked with quantum mechanics rather than ordinary mechanics, while allowing holonic transformation, such as the theory of hidden variables (that is, topological parameters in the current paper) prescribes and is currently advocated [54-60]. A useful exercise for such topo-quantum links employs the Klein bottle's "Möbius strip(s) cuttings" representation of Figure A1 (right). To it, one can assign the quaternion vector $a+b \mathbf{i}+c \mathbf{j}+$ $d \mathbf{k}$ with the following coordinates: the inside-out position (by "a", e.g., a $=0,1$, via quantum logic and, thus, the quantum information approach); position $b \in[0, I]$ along the length $(I)$ of the Klein bottle diagram; position $c \in[0, J]$ along the height $(J)$ of the Klein bottle diagram; and "the $4 \mathrm{D}$ completeness information" $d$ associated with the in-point-assignment (e.g., by $4 \mathrm{D}$ rotation algebra space or by Pauli matrices' spin algebra space) of any information contained therein, associated with the gluing diagram. Once the algebraic assignment is completed, one can pursue a finite-dimensional quaternionic quantum formalism for the description of quantum states, quantum channels, and quantum measurements [61-66]. In addition, the present results show that the next quantum 
frontier experiments should involve the nano-space dynamics of nano-tori and Klein bottle graphenic structures, and their interconnected and entangled multi-spaces; that is, Equation (6) of the main text. This corresponds with mixing topological mathematics with the theories of quantum mechanical interpretation and observations, and particularly the theory of multi-verses [30-32]. Furthermore, the current context is a mathematical and computational movement aimed at unifying the big two physical theories-namely, quantum mechanics and general relativity-in terms of space mathematics and computational confinement and dynamics, as we referred to in the recently launched project of Wolfram Research Company; see [33].

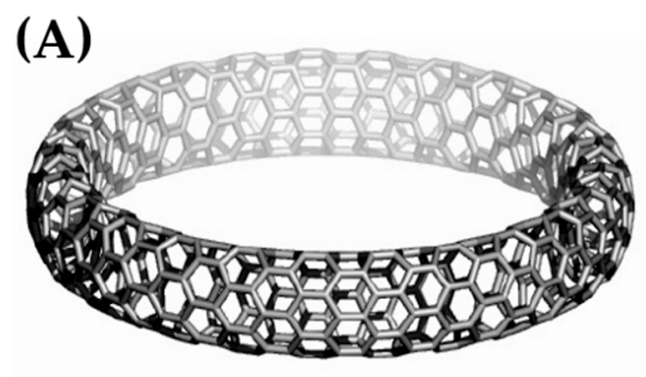

(B)
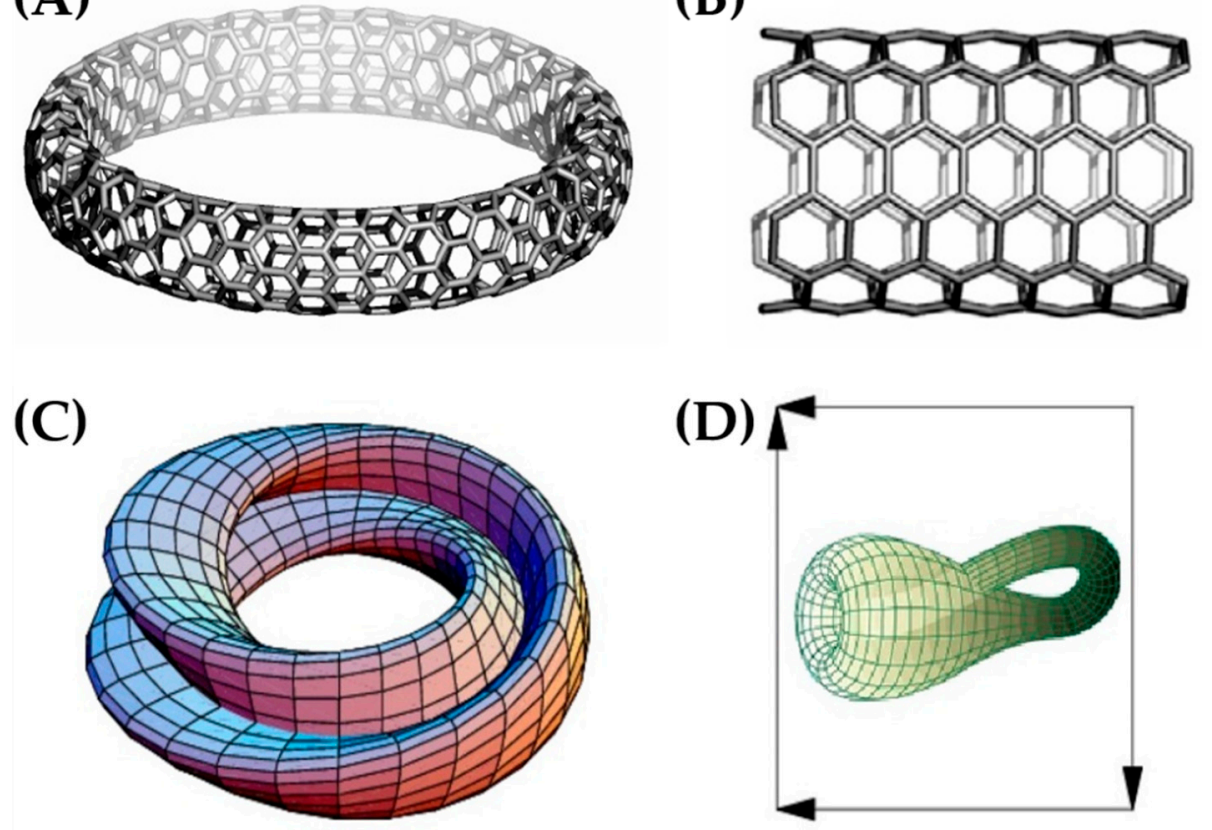

Figure A1. Upper: The $(6,3)$ covering the toroidal and cylindrical embedding, respectively. (A) $(6,3) \mathrm{H} / \mathrm{Z}$ [12,50]; $\mathrm{N}=600 ;(B) \mathrm{Tu}(6,3) \mathrm{A}[12,12] ; \mathrm{N}=144$ [49]. Bottom: The Klein bottle 3D embedding representations. (C) The so called "Figure 8 immersion"; (D) The "Möbius strip(s) cutting(s)" contained in the Klein bottle's gluing diagram-allowing its 3D representation by gluing both pairs of opposite edges, giving one pair a half-twist [2]; see the text for details.

Thus, it has been recently realized that the curved space may fit well with the associated space wave-function and, in turn, is related to the structural architecture of space. Furthermore, at the macrocosm level, such an elemental structure of space has been revealed (that is, proof of gravitational waves, presumably associated with gravitons) $[67,68]$. At the nano-cosm level, the chemical bond is far closer to the observation, measurement, experiment synthesis, and control. Nonetheless, the examination of a consistent quantum-relativistic multi-verse theory based on Klein bottle space dynamics continues in the macro-space [69]. The present work suggests that this theory is possible via nano-space shape dynamics, by the tori and Klein bottle curved degeneracies, which are both related to the graphenic (groundstate) flat-land.

\section{References}

1. Weeks, J.R. The Shape of Space; CRC Press: Boca Raton, FL, USA, 2001.

2. Weisstein, E.W. "Klein Bottle". From MathWorld-A Wolfram Web Resource. Available online: http: //mathworld.wolfram.com/KleinBottle.html (accessed on 8 May 2020).

3. Ferréol, R.; Mandonnet, J. 2017, Klein Surface, MathCurve.com. Available online: https://www.mathcurve. $\mathrm{com} /$ surfaces.gb/klein/klein.shtml (accessed on 3 March 2020). 
4. Ferréol, R.; Mandonnet, J. 2017, Genus of a Surface, MathCurve.com. Available online: https://www. mathcurve.com/surfaces.gb/genre/genre.shtml (accessed on 3 March 2020).

5. Li, P.; Zhang, Z. Continuous-time quantum walks on nonorientable surfaces: Analytical solutions for Möbius strips and Klein bottles. J. Phys. A Math. Theor. 2012, 45, 285301. [CrossRef]

6. Haruo, H. How to design non-Kekulé polyhex graphs? Croat. Chem. Acta 1986, 59, 583-590.

7. Kirby, E.C. Remarks upon recognising genus and possible shapes of chemical cages in the form of Polyhedra, Tori and Klein bottles. Croat. Chem. Acta CCACAA 1995, 68, 269-282.

8. Deza, M.; Fowler, P.W.; Rassat, A.; Rogers, K.M. Fullerenes as tilings of surfaces. J. Chem. Inf. Comput. Sci. 2000, 40, 550-558. [CrossRef]

9. Freiberger, M. Introducing the Klein bottle. Plus Magazine, 6 January 2015. Available online: https://plus.maths. org/content/introducing-klein-bottle (accessed on 15 June 2020).

10. Ori, O.; Putz, M.V. Isomeric formation of $5|8| 5$ defects in graphenic systems. Fuller. Nanotub. Carbon Nanostructures 2014, 22, 887-900. [CrossRef]

11. Ori, O.; Cataldo, F.; Putz, M.V. Topological anisotropy of Stone-Wales waves in graphenic fragments. Int. J. Mol. Sci. 2011, 12, 7934-7949. [CrossRef]

12. Ori, O.; Putz, M.V. Topological evolution of the 5| 8| 5 defect in graphene. New Front. Chem. 2018, 27, $105-113$.

13. Putz, M.V.; Ori, O.; Diudea, M.V. Bondonic electronic properties of 2D graphenic lattices with structural defects. In Graphene Science Handbook, Electrical and Optical Properties; CRC Press (Taylor \& Francis Group): Boca Raton, FL, USA, 2016; Volume 3, pp. 55-80.

14. Ori, O.; Putz, M.V.; Gutman, I.; Schwerdtfeger, P. Generalized Stone-Wales transformations for fullerene graphs derived from Berge's switching theorem. Ante Graovac Life Work. Math. Chem. Monogr. 2014, 16, 259-272.

15. Balasubramanian, K. Integration of graph theory and quantum chemistry for structure-activity relationships. SAR QSAR Environ. Res. 1994, 2, 59-77. [CrossRef]

16. Randić, M. Quantum chemical justification for Clar's valence structures. In Reviews of Modern Quantum Chemistry. A Celebration of the Contributions of the Robert G. Parr; Sen, K.D., Ed.; World Scientific: Singapore, 2002; Volume I, pp. 204-239.

17. Cataldo, F.; Ori, O.; Graovac, A. Graphene topological modifications. Int. J. Chem. Model. 2011, 3, 45.

18. Cataldo, F.; Ori, O.; Iglesias-Groth, S. Topological lattice descriptors of graphene sheets with fullerene-like nanostructures. Mol. Simul. 2010, 36, 341-353. [CrossRef]

19. Koorepazan-Moftakhar, F.; Ashrafi, A.R.; Ori, O.; Putz, M.V. Topological efficiency of fullerene. J. Comput. Theor. Nanosci. 2015, 12, 971-975. [CrossRef]

20. Deza, M.M. (At the 15th International Conference Computational and Mathematical Methods in Science and Engineering, Cadiz, Spain, 6-10 July 2015). Private Communication, 2015.

21. Sabirov, D.S.; Ori, O.; László, I. Isomers of the $\mathrm{C}_{84}$ fullerene: A theoretical consideration within energetic, structural, and topological approaches. Fuller. Nanotub. Carbon Nanostructures 2018, 26, 100-110. [CrossRef]

22. Dobrynin, A.A.; Ori, O.; Putz, M.V.; Vesnin, A.Y. Generalized topological efficiency-case study with $\mathrm{C}_{84}$ fullerene. Fuller. Nanotub. Carbon Nanostruct. 2020, 28, 545-550. [CrossRef]

23. Putz, M.V.; De Corato, M.; Benedek, G.; Sedlar, J.; Graovac, A.; Ori, O. Topological invariants of Moebius-like graphenic nanostructures. In Topological Modelling of Nanostructures and Extended Systems; Ashrafi, A.R., Cataldo, F., Iranmanesh, A., Ori, O., Eds.; Springer: Dordrecht, The Netherlands, 2013; pp. 229-244.

24. Putz, M.V. The bondons: The quantum particles of the chemical bond. Int. J. Mol. Sci. 2010, 11, 4227-4256. [CrossRef]

25. Putz, M.V.; Ori, O. Bondonic characterization of extended nanosystems: Application to graphene's nanoribbons. Chem. Phys. Lett. 2012, 548, 95-100. [CrossRef]

26. Putz, M.V.; Ori, O. Bondonic effects in Group-IV honeycomb nanoribbons with Stone-Wales topological defects. Molecules 2014, 19, 4157-4188. [CrossRef]

27. Putz, M.V.; Ori, O. Predicting bondons by Goldstone mechanism with chemical topological indices. Int. J. Quantum Chem. 2015, 115, 137-143. [CrossRef]

28. Kirby, E.C. Recent Work on Toroidal and Other Exotic Fullerenes Structures. In From Chemical Topology to Three-Dimensional Geometry; Balaban, A.T., Ed.; Kluwer Academic Publishers: New York, NY, USA, 2002; pp. 263-296. 
29. Novoselov, K.S.; Geim, A.K.; Morozov, S.V.; Jiang, D.; Zhang, Y.; Dubonos, S.V.; Grigorieva, I.V.; Firsov, A.A. Electric field effect in atomically thin carbon films. Science 2004, 306, 666-669. [CrossRef]

30. Everett, H. 'Relative State' formulation of quantum mechanics. Rev. Mod. Phys. 1957, 29, 454-462. [CrossRef]

31. Goldstein, S.; Allori, V.; Tumulka, R.; Zanghi, N. Many-worlds and Schrödinger's first quantum theory. Br. J. Philos. Sci. 2011, 62, 1-27.

32. Norsen, T. Foundations of Quantum Mechanics. An Exploration of the Physical Meaning of Quantum Theory; Springer International Publishing AG 2017: Cham, Switzerland, 2017. [CrossRef]

33. Wolfram, S. Finally We May Have a Path to the Fundamental Theory of Physics ... and It's Beautiful. Stephen Wolfram's Writings. 2020. Available online: https://writings.stephenwolfram.com/2020/04/finally-we-mayhave-a-path-to-the-fundamental-theory-of-physics-and-its-beautiful/ (accessed on 8 May 2020).

34. Kroto, H.W.; Heath, J.R.; Obrien, S.C.; Curl, R.F.; Smalley, R.E. C 60 : Buckminsterfullerene. Nature 1985, 318, 162-163. [CrossRef]

35. Geim, A.K.; Novoselov, K.S. The rise of graphene. Nat. Mater. 2007, 6, 183-191. [CrossRef]

36. Iijima, S. Helical microtubules of graphitic carbon. Nature 1991, 354, 56-58. [CrossRef]

37. Terrones, M.; Terrones, H.; Banhart, F.; Charlier, J.-C.; Ajayan, P.M. Coalescence of single-walled carbon nanotubes. Science 2000, 288, 1226-1229. [CrossRef]

38. Umemoto, K.; Saito, S.; Berber, S.; Tománek, D. Carbon foam: Spanning the phase space between graphite and diamond. Phys. Rev. B 2001, 64, 193409. [CrossRef]

39. Banhart, F. The formation of a connection between carbon nanotubes in an electron beam. Nano Lett. 2001, 1, 329-332. [CrossRef]

40. Collins, P.C.; Arnold, M.S.; Avouris, P. Engineering carbon nanotubes and nanotube circuits using electrical breakdown. Science 2001, 292, 706-709. [CrossRef]

41. Borwein, J.; Bailey, D. Mathematics by Experiment: Plausible Reasoning in the 21st Century; CRC Press: Boca Raton, FL, USA, 2008.

42. Wolfram, S. A New Kind of Science; Wolfram Media: Champaign, IL, USA, 2002; p. 1050.

43. Ori, O.; D'Mello, M. A topological study of the structure of the C76 fullerene. Chem Phys. Lett. 1992, 197, 49-54. [CrossRef]

44. Ori, O.; D'Mello, M. Analysis of the structure of the C78 fullerene: A topological approach. Appl. Phys. A 1993, 56, 35-39. [CrossRef]

45. Schwerdtfeger, P.; Wirz, L.N.; Avery, J. The topology of fullerenes. Wiley Interdiscip. Rev. Comput. Mol. Sci. 2015, 5, 96-145. [CrossRef] [PubMed]

46. Kirby, E.C.; Mallion, R.B.; Pollak, P. Toroidal polyhexes. J. Chem. Soc. Faraday Trans. 1993, 89, $1945-1953$. [CrossRef]

47. Klein, D.J. Elemental benzenoids. J. Chem. Inf. Comput. Sci. 1994, 34, 453-459. [CrossRef]

48. Ceulemans, A.; Chibotaru, L.F.; Bovin, S.A.; Fowler, P.W. The electronic structure of polyhex carbon tori. J. Chem. Phys. 2000, 112, 4271-4278. [CrossRef]

49. Diudea, M.V. (Covering Nanostructures, Faculty of Chemistry and Chemical Engineering, "Babes-Bolyai" University, 400084 Cluj, Romania). Personal Communication, 2016.

50. Reiter, K.; Weigend, F.; Wirz, L.N.; Dimitrova, M.; Sundholm, D. Magnetically induced current densities in toroidal carbon nanotubes. J. Phys. Chem. C 2019, 123, 15354-15365. [CrossRef]

51. King, R.B. Chemical Applications of topology and group theory. 29. Low density polymeric carbon allotropes based on negative curvature structures. J. Phys. Chem. 1996, 100, 15096-15104. [CrossRef]

52. King, R.B. Novel highly symmetrical trivalent graphs which lead to negative curvature carbon and boron nitride chemical structures. Disc. Math. 2002, 244, 203-210. [CrossRef]

53. Jos, L. Topology Movies. Mathematical Imagery. Available online: http://www.josleys.com/galleries.php? catid=13 (accessed on 15 June 2020).

54. Bohm, D. Wholeness and the Implicate Order; Routledge; Kegan: London, UK, 2002.

55. Rapoport, D.L. Surmounting the cartesian with philosophy, physics, logic, cybernetics and geometry: Self-reference, torsion, the Klein bottle, the time operator, multivalued logics and quantum mechanics. Found. Phys. 2011, 41, 33-76. [CrossRef]

56. Rapoport, D.L. Surmounting the cartesian cut: Klein bottle logophysics, the Dirac algebra \& the genetic code. Neuroquantoloy 2011, 9, 862-881.

57. Boeyens, J.C.A. New Theories for Chemistry; Elsevier: Amsterdam, The Netherlands, 2005. 
58. Rapoport, D.L. Torsion fields, the extended photon, quantum jumps, the Klein bottle, multivalued logic, the time operator, chronomes, perception, semiosis, neurology and cognition. In Focus in Quantum Mechanics; Hathaway, D., Randolph, E., Eds.; Nova Science: New York, NY, USA, 2011.

59. Stern, A. Quantum Theoretic Machines; Elsevier: Amsterdam, The Netherlands, 2001.

60. Rapoport, D.L. Klein bottle logophysics: A unified principle for non-linear systems, cosmology, geophysics, biology, biomechanics and perception. J. Phys. Conf. Ser. 2013, 437, 012024. [CrossRef]

61. Horwitz, L.P.; Biedenharn, L.C. Quaternion quantum mechanics: Second quantization and gauge fields. Ann. Phys. 1984, 157, 432-488. [CrossRef]

62. Adler, S.L. Quaternionic Quantum Mechanics and Quantum Fields; Oxford University Press: New York, NY, USA, 1995.

63. Hardy, L. Quantum Theory From Five Reasonable Axioms, 4th ed.Cornell University Archive: New York, NY, USA, 2001.

64. Renes, J.M.; Blume-Kohout, R.; Scott, A.J.; Caves, C.M. Symmetric informationally complete quantum measurements. J. Math. Phys. 2004, 45, 2171-2180. [CrossRef]

65. Masanes, L.; Mueller, M.P. A derivation of quantum theory from physical requirements. New J. Phys. 2001, 13, 063001. [CrossRef]

66. Wootters, W.K. Entanglement Sharing in Real-Vector-Space Quantum Theory. Found. Phys. 2012, 42, 19-28. [CrossRef]

67. Clegg, B. Gravitational Waves. How Einstein's Spacetime Ripples Reveal the Secrets of the Universe; Icon Books Ltd.: London, UK, 2018.

68. Sparrow, G. What Shape Is Space? A Primer for the 21st Century; Thames \& Hudson Ltd.: London, UK, 2018.

69. González-Díaz, P.; Alonso-Serrano, A. Observing other universe through ringholes and Klein bottle holes. Phys. Rev. D 2011, 84, 023008. [CrossRef]

(C) 2020 by the authors. Licensee MDPI, Basel, Switzerland. This article is an open access article distributed under the terms and conditions of the Creative Commons Attribution (CC BY) license (http://creativecommons.org/licenses/by/4.0/). 